\author{
P. Geisbüsch ${ }^{1}$ - J. Martin ${ }^{1}$ - E. Jenetzky² - A. Hyhlik-Dürr' • D. Kotelis ${ }^{1}$ - N. Attigah ${ }^{1}$. \\ D. Böckler ${ }^{1}$ \\ ${ }^{1}$ Klinik für Gefäßchirurgie, Vaskuläre und Endovaskuläre Chirurgie, \\ Chirurgische Universitätsklinik Heidelberg \\ 2 Institut für Medizinische Biometrie und Informatik, \\ Ruprecht-Karls-Universität Heidelberg
}

\title{
Ist Diabetes mellitus ein eigenständiger Risikofaktor in der Karotischirurgie?
}

Die Prävalenz des Diabetes mellitus liegt in der westeuropäischen Bevölkerung mittlerweile bei ca. $8 \%$. Bei den über 60-Jährigen steigt der Anteil der Diabetiker sogar auf $20 \%$ an. In großen Studien konnte bereits Ende der 1970er Jahre nachgewiesen werden, dass Diabetes mellitus ein unabhängiger Risikofaktor für Schlaganfall und Myokardinfarkt in der Bevölkerung ist $[10,20]$.

Im Rahmen elektiver gefäßchirurgischer Eingriffe, insbesondere der offenen Aneurysmachirurgie und der peripheren Bypasschirurgie weisen Patienten mit Diabetes mellitus eine erhöhte Komplikationsrate auf $[4,5,18,21,23]$. Bei der Risikostratifizierung vor Karotisthrombendarteriektomie (CEA) ist die Rolle des Diabetes mellitus hingegen in der Literatur umstritten [4, 11, 12, 15, 17, 19, 22]. Ziel dieser Studie war es deshalb, am eigenen Patientengut zu analysieren, ob Diabetes mellitus in der perioperativen Phase (30 Tage) mit einer erhöhten Schlaganfall- und Mortalitätsrate assoziiert ist und damit einen eigenständigen Risikofaktor in der Karotischirurgie darstellt.

\section{Material und Methoden}

Für den Untersuchungszeitraum von Januar 1999 bis Januar 2005 wurde eine retrospektive Analyse einer prospektiv geführten Datenbank durchgeführt. Bei $1291 \mathrm{~Pa}$ tienten (Durchschnittsalter 68,6 Jahre, $\mathrm{SD}=8,9,920$ Männer ( $71 \%)$ ) wurde wegen einer hämodynamisch relevanten extrakraniellen A.-carotis-interna-Stenose ei- ne CEA durchgeführt. Die Indikationsstellung richtete sich nach den klinischen Symptomen und dem Stenosegrad in Anlehnung an die NASCET-, ACAS- und ACST-Studienlage $[3,7,8,9]$.

Als Diabetiker wurden Patienten definiert, bei denen zum Aufnahmezeitpunkt ein Diabetes mellitus Typ I oder II bereits diagnostiziert worden war und die diätetisch bzw. medikamentös behandelt wurden. Alle Operationen wurden in Vollnarkose und unter individueller Anwendung der üblichen Rekonstruktionstechniken (Patchplastik, Eversionstechnik) durchgeführt. Die Shuntinsertion erfolgte selektiv in Abhängigkeit vom Neuromonitoring (SSEP, TCD) bzw. primär im Schlaganfallfrühstadium.

Primäre Studienendpunkte waren die perioperative ipsilaterale Schlaganfallrate und die 30-Tage-Letalität in den jeweiligen Gruppen (Diabetiker vs. Nichtdiabetiker). Sekundäre Endpunkte waren die intraoperative Komplikations- und Revisionsrate. Die statistische Auswertung erfolgte mit SPSS-Software (SPSS, Inc, Chicago/Ill). Zur statistischen Signifikanzprüfung wurde der $\chi^{2}$-Test $(\mathrm{p}<0,05)$, bzw. Mann-Whitney-U-Test $(\mathrm{p}<0,05)$ verwendet.

\section{Ergebnisse}

\section{Demografische Daten und Begleiterkrankungen}

Der Anteil der Diabetiker am Gesamtkollektiv ( $n=1291)$ betrug $28,4 \%(n=366)$ bei normal verteilter Altersstruktur in beiden Gruppen. 44,5\% der Patienten erhielten eine CEA im symptomatischen Stadium, wobei der Anteil von Patienten mit Symptomen in der Gruppe der Diabetiker nicht signifikant erhöht war (48\% Diabetiker vs. $43 \%$ Nichtdiabetiker, $p=n . s$.$) . Di-$ abetiker litten signifikant häufiger an einer arteriellen Hypertonie ( $84 \%$ vs. $77 \%$, $\mathrm{p}=0,006)$ und waren signifikant seltener aktive Raucher ( $14 \%$ vs. $20 \%, p=0,016$ ). Die übrigen kardiovaskulären Risikofaktoren waren gleich verteilt ( $\mathrm{p}=$ n.s.), wobei $57,5 \%$ des Gesamtpatientenguts 2 oder mehr kardiovaskuläre Risikofaktoren aufwiesen (• Tab. 1).

\section{Operationsergebnisse}

Die Rekonstruktionstechnik wurde in beiden Gruppen in vergleichbarer Anzahl und Verteilung durchgeführt (Eversions-TEA bei Nichtdiabtikern $78,5 \%$ vs. $70,5 \%$ bei Diabetikern). Weder der durchschnittliche Stenosegrad ( $84 \%$ vs. $85 \%$ Stenosierung), die mittlere Operationszeit (92 min vs. $94 \mathrm{~min}$ ) noch die mittlere Abklemmdauer (22 min vs. $24 \mathrm{~min}$ ) waren in den Gruppen signifikant unterschiedlich. Bei Diabetikern wurde signifikant häufiger ein temporärer Shunt eingelegt (32\% vs. $24 \%$, p=0,002, OR 1,535, 95\%-CI 1,1772,001). Die intraoperative Komplikationsrate $(6,8 \%$ vs. $6,1 \%)$ sowie die Revisionsrate $(2,7 \%$ vs. $3,5 \%)$ wiesen in beiden Gruppen keinen Unterschied auf ( $\mathrm{p}=0,356$ bzw. $\mathrm{p}=0,756)(\bullet$ Tab. 2). 
Perioperative Schlaganfallund Mortalitätsrate

Das Risiko eines perioperativen Schlaganfalls war bei Diabetikern nicht signifikant erhöht und betrug für alle Stadien bei Diabetikern 3,8\% ( $n=14 / 366)$ und bei Nichtdiabetikern 2,8\% (25/925) $(p=0,343)$. Die perioperativen Schlaganfallursachen zeigt $\bullet$ Tab. 3. Die 30-TageLetalität betrug im Gesamtkollektiv 0,54\% $(\mathrm{n}=7 / 1291)$ und unterschied sich bei Diabetikern $0,27 \%(n=1)$ und bei Nichtdiabetikern $0,62 \%(n=6)$ nicht signifikant $(p=0,408)$. Zerebrale Ischämien und Einblutungen sowie kardiopulmonale Komplikationen bildeten hierbei die Haupttodesursachen (• Tab. 4). Die kombinierte Schlaganfall- und Mortalitätsrate betrugt $3,4 \%$ bei Diabetikern und $4,1 \%$ bei Nichtdiabetikern ohne signifikanten Unterschied $(p=0,584)$.

\section{Diskussion}

Im eigenen Patientengut konnte die vorgestellte Studie zeigen, dass Diabetes mellitus kein unabhängiger Risikofaktor in der Karotischirurgie in Bezug auf perioperative Schlaganfall- und Todesrate ist. Signifikant häufiger musste bei pathologischem Neuromonitoring bei Diabetikern ein temporärer Shunt eingebracht werden.

Bei den großen randomisierten Studien zur Effizienz der Schlaganfalltherapie mittels Karotisendarteriektomie (CEA) liegt der Anteil der Diabetiker zwischen $11,7 \%$ (ESCET), 21,6\% (NASCET) und $23 \%$ (ACAS). Er liegt somit etwas unterhalb des eigenen, hier vorgestellten Kollektivs mit $28,4 \%[3,16]$. In der Literatur herrscht derzeit über die Frage, inwieweit Diabetes mellitus einen eigenständigen Risikofaktor in der Karotischirurgie darstellt, kein Konsens. Während einige Studien auf eine erhöhte Schlaganfallund Komplikationsrate nach Karotis-TEA bei Diabetikern verweisen, sehen andere Autoren Diabetes mellitus nicht als eigenständigen Risikofaktor der operativen Therapie extrakranieller Karotisstenosen an (- Tab. 5) $[1,11,15,19]$. Ahari et al. [1] zeigten bei 2622 Patienten und einem Diabetikeranteil von $13 \%$ eine signifikant erhöhte 30 -Tage-Letalität (3,2\% vs. $1,4 \%$ $\mathrm{p}=0,02)$ und 1-Jahres-Mortalität (7,9\% vs.

P. Geisbüsch · J. Martin · E. Jenetzky · A. Hyhlik-Dürr · D. Kotelis · N. Attigah · D. Böckler Ist Diabetes mellitus ein eigenständiger Risikofaktor in der Karotischirurgie?

\section{Zusammenfassung}

Hintergrund. Der Einfluss des Diabetes mellitus in der Risikostratifizierung der Karotisthrombendarteriektomie (CEA) wird in der Literatur kontrovers diskutiert. Ziel dieser Single-Center-Studie ist der Vergleich der perioperativen Schlaganfall- und Todesrate bei Diabetikern und Nichtdiabetiker nach CEA zur Beantwortung der Frage, inwieweit Diabetes mellitus hierbei einen eigenständigen Risikofaktor darstellt.

Material und Methoden. Es erfolgte eine retrospektive Analyse von 1291 Patienten (Durchschnittsalter 68,6 Jahre, $S D=8,9$, 920 Männer), bei denen in einem 6-jährigen Zeitraum (01/1999-01/2005) wegen einer asymptomatischen oder symptomatischen extrakraniellen Karotisstenose eine CEA durchgeführt wurde. Primäre Studienendpunkte waren die perioperative ipsilaterale Schlaganfallrate und 30-Tage-Letalität in beiden Gruppen (Diabetiker vs. Nichtdiabetiker). Sekundäre Endpunkte waren intraoperative Komplikations- und Revisionsrate. Ergebnisse. Der Anteil der Diabetiker am Gesamtkollektiv betrug 28,4\% ( $n=366$ ). Der Anteil von Patienten mit Symptomen war in beiden Gruppen (48\% Diabetiker vs. $43 \%$ Nichtdiabetiker) gleich verteilt. Beide Gruppen waren hinsichtlich der Rekonstruktionstechnik (Eversion vs. Patchplastik) dem durchschnittlichem Stenosegrad, der Operationszeit und Abklemmdauer vergleichbar. Bei Diabetikern wurde signifikant häufiger ein temporärer Shunt eingelegt (32\% vs. $24 \%, p=0,002$, OR $1,535,95 \%$-Cl 1,177$2,001)$. Alle Operationen wurden in Vollnarkose durchgeführt. Die perioperative Schlaganfallrate betrug im Gesamtkollektiv 3,0\%, bei Diabetikern 3,8\% ( $n=14 / 366)$ und bei Nichtdiabetikern $2,8 \%(25 / 925)(p=0,343)$. Die 30 -Tage-Letalität betrug insgesamt 0,54\% ( $n=7 / 1291)$, bei Diabetikern 0,27\% $(n=1)$ und bei Nichtdiabetikern $0,62 \%(n=6)$ ohne signifikanten Unterschied $(p=0,408)$. Die kombinierte Apoplex- und Mortalitätsrate beträgt somit 3,4\% (Diabetiker) bzw. 4,1\% (Nichtdiabetiker) $(p=0,584)$. Die intraoperative Komplikationsrate sowie die Revisionsrate wiesen in beiden Gruppen keinen Unterschied auf ( $p=0,356$ bzw. $p=0,756$ )

Schlussfolgerung. Im eigenen Patientengut ist Diabetes mellitus kein eigenständiger Risikofaktor für den perioperativen Schlaganfall und den Tod jeglicher Ursache. Diabetiker benötigten signifikant häufiger eine temporäre Shuntinsertion.

Schlüsselwörter

Diabetes mellitus · CEA · Karotischirurgie · Karotisthrombendarteriektomie $\cdot$ Schlaganfall

\section{Is diabetes mellitus an independent risk factor for adverse events after carotid endarterectomy?}

\section{Abstract}

Background. The status of diabetes mellitus as a predictor of poor outcome after carotid endarterectomy (CEA) is controversial. The objective of this study was to evaluate and compare the perioperative death and stroke rates after CEA in patients with and without diabetes.

Material and methods. A retrospective analysis of 1,291 patients (mean age 68.6 years, SD 8.9 years; 920 men) undergoing CEA for carotid artery stenosis between January 1999 and January 2005 was performed.

Results. Of this study population, $28.4 \%$ $(n=366)$ were diabetic. The same proportion of symptomatic patients was seen in both groups ( $48 \%$ diabetics vs. $43 \%$ nondiabetics; not significant). No differences regarding surgical technique, degree of stenosis, operating time, or clamping time were observed. Selective shunting was used significantly more often in diabetics (32\%) than in nondiabetics
( $24 \% ; p=0.002$, odds ratio $1.535,95 \%$ confidence interval 1.177-2.001). The perioperative stroke rate in diabetic patients was $3.8 \%$ ( $n=14 / 366)$ compared to $2.8 \%(25 / 925)$ in nondiabetic patients $(p=0.343)$. The overall mortality was $0.54 \%(n=7 / 1,291) ; 0.27 \%$ $(n=1)$ in diabetics and $0.62 \%(n=6)$ in nondiabetics $(p=0.408)$. The combined death and stroke rate was $3.4 \%$ in diabetics versus $4.1 \%$ in nondiabetics ( $p=0.584)$.

Conclusion. In this study, the presence of diabetes did not influence perioperative stroke and death rates after CEA. Shunt insertion was significantly more frequent in diabetic patients.

Keywords

Diabetes mellitus - CEA - Carotid surgery Carotid endarterectomy $\cdot$ Stroke 


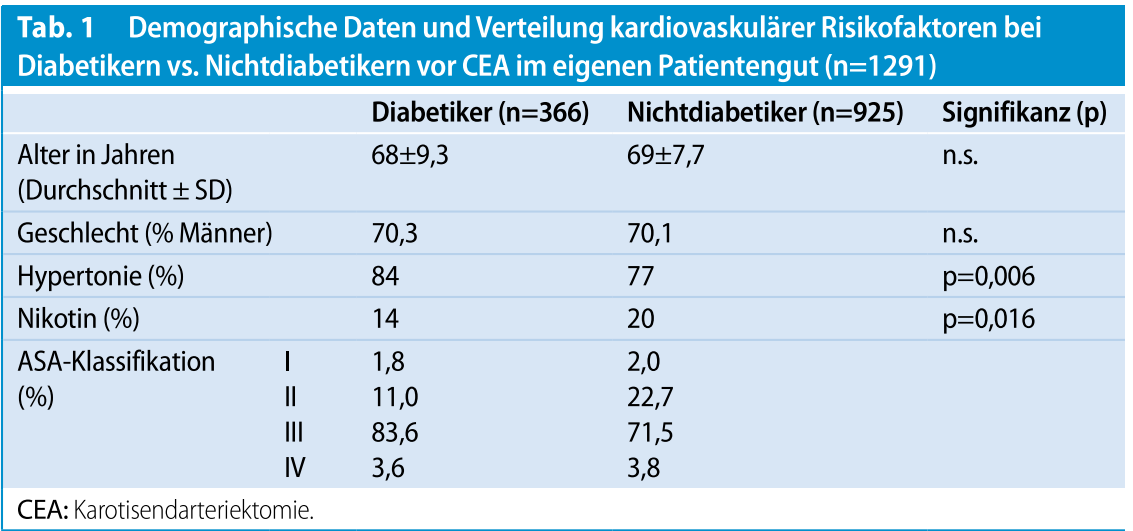

Tab. 2 Operationsindikation und Ergebnisse bei Diabetikern vs. Nichtdiabetikern nach CEA im eigenen Patientengut ( $n=1291$ )

\begin{tabular}{|c|c|c|c|}
\hline & Diabetiker $(n=366)$ & Nichtdiabetiker $(n=925)$ & Signifikanz (p) \\
\hline $\begin{array}{l}\text { Anteil symptomatischer ACl- } \\
\text { Stenosen (\%) }\end{array}$ & 48 & 43 & n.s. \\
\hline $\begin{array}{l}\text { Stenosegrad in \% } \\
\text { (Durchschnitt } \pm \text { SD) }\end{array}$ & $85 \pm 10.1$ & $84 \pm 11,5$ & n.s. \\
\hline $\begin{array}{l}\text { Operationszeit in min } \\
\text { (Durchschnitt } \pm S D \text { ) }\end{array}$ & $94 \pm 28,7$ & $92 \pm 36,7$ & n.s. \\
\hline Mittlere Abklemmzeit (min) & 24 & 22 & n.s. \\
\hline Anteil der Eversions-TEA (\%) & 71 & 79 & n.s. \\
\hline Shuntinsertationsrate (\%) & 32 & 24 & $p=0,002$ \\
\hline $\begin{array}{l}\text { Perioperative Komplikations- } \\
\text { rate (\%) }\end{array}$ & 6,8 & 6,1 & n.s. \\
\hline Revisionsrate (\%) & 2,7 & 3,5 & n.s. \\
\hline Hirnnervenläsionen (\%) & 1,5 & 1,2 & n.s. \\
\hline Schlaganfallrate (\%) & 3,8 & 2,8 & n.s. \\
\hline 30-Tage-Letalität (\%) & 0,27 & 0,62 & n.s. \\
\hline \multicolumn{4}{|c|}{ CEA: Karotisendarteriektomie; n.s.: nicht signifikant. } \\
\hline
\end{tabular}

Tab. 3 Perioperative Schlaganfallursachen nach CEA im eigenen Patientengut ( $n=39 / 1291)$

\begin{tabular}{|c|c|c|c|}
\hline Apoplexursache & Diabetiker $(n=366)$ & Nichtdiabetiker $(n=925)$ & Signifikanz (p) \\
\hline Intraoperative Embolie & 3 & 6 & \\
\hline HIT II & 2 & 4 & \\
\hline Clamping & & 2 & \\
\hline ICB & & 2 & \\
\hline $\begin{array}{l}\text { Akuter thrombotischer } \\
\text { Verschluss }\end{array}$ & & 3 & \\
\hline Unklar & 9 & 8 & \\
\hline Gesamt & $14 / 366=3,8 \%$ & $25 / 925=2,8 \%$ & $p=0,584$ \\
\hline
\end{tabular}

4,4\% $\mathrm{p}=0,008$ ) bei Diabetikern nach Karotis-TEA, wobei kardiale Komplikationen die Haupttodesursache darstellten. Hinsichtlich neurologischer Komplikationen zeigte sich jedoch kein signifikanter Unterschied in beiden Patientengruppen, was sich mit unseren Ergebnissen deckt. Im Gegensatz zu diesen Ergebnissen beschrieben Tu et al. [19] in ihrem Patientenkollektiv von $n=6038$ Patienten Dia- hängige Risikoprädiktoren für eine signifikant erhöhte Morbidität und Mortalität. Analog hierzu konnten Kragsterman et al. [11] in ihrer Serie von $n=1518$ CEA Diabetes mellitus als eigenständigen Risikofaktor (relatives Risiko 1,41; 95\%-CI: 1,04$1,89, p=0,0002$ ) einer erhöhten kombinierten Schlaganfall- und Todesrate aufzeigen.

In Übereinstimmung mit den eigenen vorgestellten Ergebnissen findet sich in weiteren Studien kein erhöhtes Risiko für Diabetiker nach Karotis-TEA [2, 13, 15]. Akbari et al. [2] berichten in Ihrem Kollektiv von 732 CEA bei einem hohen Diabetikeranteil von $39 \%$ über eine vergleichbare neurologische Komplikationsrate von $1,0 \%$ vs. $1,1 \%$; p>0,05) und über eine Mortalitätsrate von $0,35 \%$ vs. $0,2 \%$ ( $p>0,05)$ in beiden Guppen. Ähnliche Ergebnisse wurden von Pistolese et al. [13] publiziert, die in ihrer Serie von 781 CEA (DM: 24,7\%, $n=193$ ) ebenfalls keinen Unterschied im perioperativem Ergebnis (Apoplex, Myokardinfarkt, Mortalität) und 5-Jahres-Überleben nach CEA in beiden Untergruppen finden konnten. Diabetes mellitus korrelierte in dieser Studie jedoch interessanterweise mit einer signifikant erhöhten Plaqueausdehnung (Plaquelänge) und damit mit einer längeren Arteriotomie und Klemmzeit. Aufgrund des retrospektiven Studiendesigns und der nicht durchgeführten Plaqueanalyse dieser Studie können die Beobachtungen von Pistolese nicht reproduziert werden. Eine klinisch-praktische Konsequenz ist daraus nicht unmittelbar abzuleiten. Im eigenen Patientengut zeigte sich bei Diabetes mellitus keine verlängerte Klemm- und Operationszeit als potenziell indirekter Hinweis auf eine eventuell vermehrte Plaqueausdehnung und damit ggf. zeitaufwendigere Rekonstruktionstechnik. Hinsichtlich des Langzeitverlaufs nach CEA zeigten Ballotta et al. [6] neben einer vergleichbaren neurologischen Komplikations- und Mortalitätsrate ebenfalls keinen Unterschied bezüglich Restenose oder Verschlussrate $(\mathrm{n}=547$; Diabetes mellitus $36,4 \%$; durchschnittliche Followup-Zeit: 44 Monate; Spanne 1-75 Monate) in beiden Gruppen.

Rockman et al. [15] publizierten in einer retrospektiven Studie von 2151 Patienten (507 Diabetiker vs. 1644 Nichtdiabe- 
tiker) keine erhöhte perioperative Morbidität ( $0,6 \%$ vs. $0,4 \%, p=$ n.s.) und Letalität (o,8\% vs. $0,5 \% ; \mathrm{p}=$ n.s.), insbesondere keine erhöhte perioperative Apoplexrate $(3,2 \%$ vs $2,4 \% ; p=n$.s.) für Diabetiker. Diese Ergebnisse erscheinen umso bemerkenswerter, als Diabetiker in dieser Serie signifikant häufiger unter einer arteriellen Hypertonie und/oder KHK litten, sowie signifikant häufiger $(52,5 \%$ vs. $47,1 \%$; $=0,04)$ im symptomatischen Stadium operiert wurden. Auch in unserem Patientengut lag eine arterielle Hypertonie bei Diabetikern signifikant häufiger vor. Kontralateraler Karotisverschluss, Klemmtoleranz und Shuntinsertion stellten nach Rockman unabhängige Risikofaktoren für den perioperativen Tod dar. Im eigenen Patientengut hatten Diabetiker trotz signifikant häufigerer Shuntinsertion und häufigerem arteriellen Hypertonus kein erhöhtes perioperatives Schlaganfall- und Todesfallrisiko.

Eine Analyse perioperativer Apoplexursachen bei $n=3062$ CEA (Diabetes mellitus: $20 \%$ ) aus derselben Arbeitsgruppe konnte technische Fehler in der Subgruppe der Diabetiker analog zu unseren Ergebnissen ebenfalls als Hauptschlaganfallquelle ausmachen [14].

Die Analyse unseres Patientenguts konnte Diabetes mellitus als eigenständigen Risikofaktor in der Karotischirurgie ebenfalls ausschließen und unterstreicht somit, bei vergleichbarer perioperativer Morbidität und Mortalität, die Ergebnisse von Rockman et al. [15].

Schwachpunkte der vorgestellten Studie liegen in Ihrem retrospektiven Charakter und einem nicht auszuschließenden Bias durch weitere nicht berücksichtigte Faktoren wie z. B. den Ausbildungsstand der Operateure oder der Plaquemorphologie.

\section{Fazit für die Praxis}

Im eigenen Patientengut stellt Diabetes mellitus keinen eigenständigen Risikofaktor für die operative Schlaganfallprophylaxe durch CEA dar. Somit hat das Vorliegen von Diabetes mellitus in unserer Klinik keinen Einfluss auf die Patientenselektion hinsichtlich Indikation, Operationstechnik und postoperativem intensivmedizinischem Überwachungszeitraum. Auffällig ist die höhere Inzidenz

Tab. 4 30-Tage-Letalität nach Karotisendarteriektomie ( $n=1291)$

\begin{tabular}{|c|c|c|c|}
\hline Todesursache & Diabetiker $(n=366)$ & Nichtdiabetiker $(n=925)$ & Signifikanz (p) \\
\hline $\begin{array}{l}\text { Sepsis } \\
\text { (nicht assoziiert) }\end{array}$ & & 2 & \\
\hline Zerebrale Hämorrhagie & & 2 & \\
\hline Kardiopulmonales Versagen & 1 & 1 & \\
\hline Ischämischer Infarkt & & 1 & \\
\hline Gesamt 7/1291 (0,54\%) & $1 / 366(0,27 \%)$ & $6 / 925(0,62 \%)$ & $p=0,408$ \\
\hline
\end{tabular}

Tab. 5 Ausgewählte Literaturübersicht mit Prävalenz des Diabetes mellitus im Rahmen der Karotischirurgie sowie Ergebnisse im Vergleich von Diabetikern mit Nichtdiabetikern

\begin{tabular}{|c|c|c|c|c|c|}
\hline \multirow[t]{3}{*}{ Studie } & CEA & Diabetiker & Apoplexrate & 30-Tage-Letalität & $\begin{array}{l}\text { Kombinierte } \\
\text { Schlaganfall-/To- } \\
\text { desrate }\end{array}$ \\
\hline & & & \multicolumn{3}{|c|}{ Diabetiker vs. Nichtdiabetiker } \\
\hline & (n) & (\%) & (\%) & (\%) & (\%) \\
\hline $\begin{array}{l}\text { Rockman [15], } \\
2005\end{array}$ & 2151 & 23,6 & $\begin{array}{l}3,2 \text { vs. } 2,4 \\
\text { n.s. }\end{array}$ & $\begin{array}{l}0,8 \text { vs. } 0,5 \\
\text { n.s. }\end{array}$ & k.A. \\
\hline \multirow{3}{*}{$\begin{array}{l}\text { Kragsterman [11] } \\
(2004)\end{array}$} & \multirow[t]{3}{*}{1518} & 13,8 & \multicolumn{3}{|c|}{ Diabetiker und Nichtdiabetiker } \\
\hline & & & 3,3 & 1,4 & 4,3 \\
\hline & & & n.s. & $\begin{array}{l}\text { RR 2,03 (95\%-Cl: } \\
1,29-3,21) \\
(p=0,002)\end{array}$ & $\begin{array}{l}\text { RR 1,41 (95\%-Cl: } \\
1,04-1,89) \\
(p=0,025)\end{array}$ \\
\hline \multirow[t]{3}{*}{ Tu [19] (2003) } & 6038 & 23,1 & \multicolumn{3}{|c|}{ Diabetiker vs. Nichtdiabetiker } \\
\hline & & & 5,0 vs. 4,5 & 2,6 vs. 1,6 & 7,4 vs. 6,0 \\
\hline & & & n.s. & $\begin{array}{l}\text { OR 2,13 (95\%-Cl: } \\
1,39-3,26) \\
(p=0,0005)\end{array}$ & $\begin{array}{l}\text { OR } 1,28(95 \%-C l: \\
1,01-1,63) \\
(p=0,043)\end{array}$ \\
\hline $\begin{array}{l}\text { Pistolese [13] } \\
(2001)\end{array}$ & 781 & 24,7 & $\begin{array}{l}1,5 \text { vs. } 0,5 \\
\text { n.s }\end{array}$ & $\begin{array}{l}1,5 \text { vs. } 0,5 \\
\text { n.s }\end{array}$ & k.A. \\
\hline Ballotta [6] (2000) & 547 & 36,4 & $\begin{array}{l}1,0 \text { vs. } 2,3 \\
\text { n.s. }\end{array}$ & $\begin{array}{l}0,5 \text { vs. } 0,5 \\
\text { n.s. }\end{array}$ & k.A \\
\hline Ahari [1] (1999) & 2622 & 13 & $\begin{array}{l}3,5 \text { vs. } 2,8 \\
\text { n.s. }\end{array}$ & $\begin{array}{l}3,2 \text { vs. } 1,4 \\
(p=0,02)\end{array}$ & $\begin{array}{l}6,7 \text { vs. } 4,1 \\
\text { univariante Analy- } \\
\text { se }(p=0,035) \text { mul- } \\
\text { tivariante Analyse } \\
(p=0,07)\end{array}$ \\
\hline Akbari [2] (1997) & 732 & 39 & $\begin{array}{l}1,0 \text { vs. } 1,1 \\
\text { n.s. }\end{array}$ & $\begin{array}{l}0,35 \text { vs. } 0,2 \\
\text { n.s. }\end{array}$ & k.A. \\
\hline Eigene Serie & 1291 & 28,4 & $\begin{array}{l}3,8 \text { vs. } 2,8 \\
\text { n.s. }\end{array}$ & $\begin{array}{l}0,27 \text { vs. } 0,62 \\
\text { n.s. }\end{array}$ & $\begin{array}{l}3,4 \text { vs. } 4,1 \\
\text { n.s. }\end{array}$ \\
\hline
\end{tabular}

CEA: Karotisendarteriektomie; k.A.: keine Angabe; n.s.: nicht signifikant; RR: relatives Risiko; OR: Odds-Ratio.

von Shuntinsertionen bei Diabetikern. Ob Diabetes mellitus einen Risikofaktor beim Karotisstenting darstellt, muss durch Subgruppenanalysen der randomisierten Studien beantwortet werden.

\section{Korrespondenzadresse} Dr. P. Geisbüsch

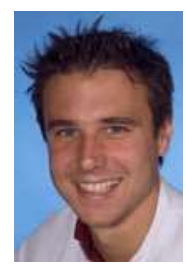

Klinik für Gefäßchirurgie Vaskuläre und Endovaskuläre Chirurgie, Chirurgische Universitätsklinik Heidelberg Im Neuenheimer Feld 110, 69120 Heidelberg philipp.geisbuesch@med.uniheidelberg.de
Interessenkonflikt. Der korrespondierende Autor gibt an, dass kein Interessenkonflikt besteht.

\section{Literatur}

1. Ahari A, Bergqvist D, Troeng T et al. (1999) Diabetes mellitus as a risk factor for early outcome after carotid endarterectomy-a population-based study. Eur JVasc Endovasc Surg 18: 122-126

2. Akbari CM, Pomposelli FB Jr, Gibbons GW et al. (1997) Diabetes mellitus: A risk factor for carotid endarterectomy? J Vasc Surg 25: 1070-1075; discussion 1075-1076

3. Anonymous (1995) Endarterectomy for asymptomatic carotid artery stenosis. Executive Committee for the Asymptomatic Carotid Atherosclerosis Study. JAMA 273: 1421-1428 
4. Axelrod DA, Upchurch GR Jr, DeMonner S et al. (2002) Perioperative cardiovascular risk stratification of patients with diabetes who undergo elective major vascular surgery. J Vasc Surg 35: 894901

5. Aziz I, Lewis RJ, Baker JD et al. (2001) Cardiac morbidity and mortality following carotid endarterectomy: the importance of diabetes and multiple Eagle risk factors. Ann Vasc Surg 15: 243-246

6. Ballotta E, Da Giau G, Renon L (2001) Is diabetes mellitus a risk factor for carotid endarterectomy? A prospective study. Surgery 129: 146-152

7. Barnett HJ, Taylor DW, Eliasziw M et al. (1998) Benefit of carotid endarterectomy in patients with symptomatic moderate or severe stenosis. North American Symptomatic Carotid Endarterectomy Trial Collaborators. N Engl J Med 339: 1415-1425

8. Eckstein HH (2004) Surgical therapy of extracranial carotid stenosis. Chirurg 75: 93-110

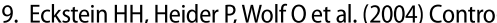
versies in the treatment of carotid stenoses. Present state of research and evidence-based medicine. Chirurg 75: 672-680

10. Kannel WB, McGee DL (1979) Diabetes and cardiovascular disease. The Framingham study. JAMA 241: 2035-2038
11. Kragsterman B, Logason $K$, Ahari A et al. (2004) Risk factors for complications after carotid endarterectomy - a population-based study. Eur J Vasc Endovasc Surg 28: 98-103

12. LaMuraglia GM, Brewster DC, Moncure AC et al. (2004) Carotid endarterectomy at the millennium: what interventional therapy must match. Ann Surg 240: 535-544; discussion 544-546

13. Pistolese GR, Appolloni A, Ronchey S et al. (2001) Carotid endarterectomy in diabetic patients. JVasc Surg 33: 148-154

14. Riles TS, Imparato AM, Jacobowitz GR et al. (1994) The cause of perioperative stroke after carotid endarterectomy. J Vasc Surg 19: 206-214; discussion 215-216

15. Rockman CB, Saltzberg SS, Maldonado TS et al (2005) The safety of carotid endarterectomy in diabetic patients: clinical predictors of adverse outcome. JVasc Surg 42: 878-883

16. Rothwell PM, Eliasziw M, Gutnikov SA et al. (2004) Endarterectomy for symptomatic carotid stenosis in relation to clinical subgroups and timing of surgery. Lancet 363: 915-924

17. Salenius JP, Harju E, Riekkinen H (1990) Early cerebral complications in carotid endarterectomy: risk factors. J Cardiovasc Surg (Torino) 31: 162-167
18. Treiman GS, Treiman RL, Foran RF et al. (1994) The influence of diabetes mellitus on the risk of abdominal aortic surgery. Am Surg 60: 436-440

19. Tu JV, Wang H, Bowyer B et al. (2003) Risk factors for death or stroke after carotid endarterectomy: observations from the Ontario Carotid Endarterectomy Registry. Stroke 34: 2568-2573

20. Tuomilehto J, Rastenyte D, Jousilahti P et al. (1996) Diabetes mellitus as a risk factor for death from stroke. Prospective study of the middle-aged Finnish population. Stroke 27: 210-215

21. Virkkunen J, Heikkinen $M$, Lepantalo $M$ et al. (2004) Diabetes as an independent risk factor for early postoperative complications in critical limb ischemia. JVasc Surg 40: 761-767

22. Weiss JS, Sumpio BE (2006) Review of prevalence and outcome of vascular disease in patients with diabetes mellitus. Eur J Vasc Endovasc Surg 31: 143-150

23. Yeager RA, Moneta GL, McConnell DB et al. (1989) Analysis of risk factors for myocardial infarction following carotid endarterectomy. Arch Surg 124 1142-1145 